\title{
Identifying optimal candidates for primary tumor resection among metastatic non-small cell lung cancer patients: a population-based predictive model
}

\author{
Hengrui Liang ${ }^{1 \#}$, Zhichao Liu ${ }^{2,3 \#}$, Jun Huang ${ }^{1 \#}$, Jun Liu ${ }^{1}$, Wei Wang ${ }^{1}$, Jianfu Li ${ }^{1}$, Shan Xiong ${ }^{1}$, Caichen Li ${ }^{1}$, \\ Bo Cheng ${ }^{1}$, Yi Zhao ${ }^{1}$, Fei Cui ${ }^{1}$, Jianxing $\mathrm{He}^{1}$, Wenhua Liang ${ }^{1}$ \\ ${ }^{1}$ Department of Thoracic Surgery and Oncology, The First Affiliated Hospital of Guangzhou Medical University, State Key Laboratory of \\ Respiratory Disease, National Clinical Research Center for Respiratory Disease, Guangzhou Institute of Respiratory Health, Guangzhou, China; \\ ${ }^{2}$ Nanshan School, Guangzhou Medical University, Guangzhou, China; ${ }^{3}$ Department of Thoracic Surgery, Shanghai Chest Hospital, Shanghai Jiao \\ Tong University, Shanghai, China \\ Contributions: (I) Conception and design: All authors; (II) Administrative support: J He, W Liang; (III) Provision of study materials or patients: H \\ Liang, Z Liu; (IV) Collection and assembly of data: H Liang, Z Liu, J Huang; (V) Data analysis and interpretation: All authors; (VI) Manuscript \\ writing: All authors; (VII) Final approval of manuscript: All authors. \\ "These authors contributed equally to this work. \\ Correspondence to: Fei Cui; Jianxing He; Wenhua Liang. Department of Thoracic Surgery and Oncology, The First Affiliated Hospital of Guangzhou \\ Medical University, Guangzhou 510120, China; State Key Laboratory of Respiratory Disease, Guangzhou 510120, China; National Clinical \\ Research Center for Respiratory Disease, Guangzhou 510120, China; Guangzhou Institute of Respiratory Health, Guangzhou 510120, China. \\ Email: cuidavil@hotmail.com; drjianxing.he@gmail.com; liangwh1987@163.com.
}

Background: A survival benefit was observed in metastatic non-small cell lung cancer (NSCLC) patients that underwent surgical resection of the primary tumor. We developed a model testing the hypothesis that only certain stage IV patients would benefit from surgery and the potential benefit would vary based on primary tumor characteristics.

Methods: Patients with stage IV NSCLC were identified in the Surveillance, Epidemiology and End Results (SEER) database and then divided into surgery and non-surgery groups. A 1:1 Propensity score matching (PSM) was performed to balance characters. We assumed that patients received primary tumor surgery that lived longer than median cancer specific survival (CSS) time of those who didn't underwent surgery could benefit from the operation. Multivariable Cox model was used to explore the independent factors of CSS in two groups (beneficial and non-beneficial group). Logistic regression was used to build a nomogram based on the significant predictive factors.

Results: A total of 30,342 patients with stage IV NSCLC were identified; $8.03 \%(2,436)$ received primary tumor surgery. After PSM, surgical intervention was independently correlated with longer median CSS time (19 vs. 9 months, $\mathrm{P}<0.001)$. Among the surgery cohort, 1,374 (56.40\%) patients lived longer than 9 months (beneficial group). Differentiated characters (beneficial and non-beneficial group) included age, gender, TNM stage, histologic type, tumor position and differentiation grade, which were integrated as predictors to build a nomogram.

Conclusions: A practical predictive model was created and might be used to identify the optimal candidates for surgical resection of the primary tumor among stage IV NSCLC patients.

Keywords: Stage IV non-small cell lung cancer; surgery; predictive model; SEER database; nomogram

Submitted Jun 01, 2020. Accepted for publication Oct 06, 2020.

doi: $10.21037 /$ tlcr-20-709

View this article at: http://dx.doi.org/10.21037/tlcr-20-709 


\section{Introduction}

Lung cancer is one of the most common cancers worldwide as well as the leading cause of cancer death (1). Non-small cell lung cancer (NSCLC) comprises over $80 \%$ of lung cancer cases (2). Approximately half of all NSCLC patients present with metastatic disease at diagnosis, therapeutic scheme are rather limited and life expectancy is greatly compromised. In these patients, chemotherapy, targeted therapy and immunotherapy represent the primary choice of treatment (3).

Local resection to primary tumor site is not usually taken into account as conventional treatment in stage IV NSCLC patients (4). The primary focus and goals in such patients is disease control and palliation but not radical cure (5). Many studies have demonstrated the primary tumor resection was associated with improved survival outcomes. However, surgical intervention whether to primary or metastatic tumor site for advanced-stage NSCLC has been proved to be associated with improvements in survival (68 ) in advanced NSCLC patients $(9,10)$, especially in M1a patients without N3 or pericardial effusion disease (10). From these studies, it can be concluded that not all stage IV patients prolong their lives from primary tumor surgery, the specific group of patients who do benefit from the surgery are still not fully understood.

We aimed to develop a predictive model using a prospective national database to test the hypothesis that only certain patients would benefit from surgery and that the potential benefit would vary depend on primary tumor characteristics. We present the following article in accordance with the Transparent Reporting of a multivariable prediction model for Individual Prognosis Or Diagnosis (TRIPOD) reporting checklist (11) (available at http://dx.doi.org/10.21037/tlcr-20-709).

\section{Methods}

\section{Patient selection in SEER database}

The Surveillance, Epidemiology and End Results (SEER) database (1973-2014, November 2016 submission) is a national population-based reporting system that collects tumor-related data, including the incidence, treatment, mortality, and other demographics, covering approximately $28 \%$ of the US population (12). The SEER data contain no identifiers and are publicly available for studies of cancerbased epidemiology and survival analysis. We had got the permission to access these research data (SEER-Stat username: lianghr). The study was conducted in accordance with the Declaration of Helsinki (as revised in 2013).

Cases of lung cancer (C34.0-34.9) diagnosed from 2004 to 2014 were extracted from the SEER database (SEERStat 8.3.5) according to the site code classifications. We selected this range because American Joint Committee on Cancer (AJCC) TMN stage and Collaborative Stage (CS) information was available since 2004 and patients diagnosed after 2014 were excluded to ensure an adequate follow-up time. We reclassified the TNM stage according to AJCC $8^{\text {th }}$ edition. Patients diagnosed as stage IV and histologically confirmed as NSCLC were enrolled. Patients were excluded if the surgery to primary site record was unknown. Other exclusion criteria were as follows: less than 18 years old, unknown TNM stage, unknown survival months, unknown treatment modality, not the first tumor and not only one tumor.

\section{Statistical analysis}

The study sample was divided according to treatment, surgery versus non-surgery treatment of the primary tumor. Propensity-score matching (PSM) was calculated to account for confounding variables and facilitate matching patients in the two treatment groups ( $\mathrm{R}$ software version 2.15.1, https://www.rproject.org/). Variables that could influence the outcomes of treatment were used to generate a propensity score by logistic regression, including age, gender, TNM stage, differentiation grade, primary site, laterality, histological type, radiation therapy, chemotherapy and surgery to metastasis site. Patients in the two groups (surgery and non-surgery) were 1:1 matched using the nearest propensity score on the logit scale. The caliper was set at 0.01 . After PSM, differences in continuous (age) and categorical clinical characteristics (others except for age) were tested for significance by student $t$ test and chi-square tests, respectively.

Overall survival (OS), cancer specific survival (CSS) and survival month were extracted from SEER database. OS was the time from diagnosis to death from any cause; living patients were excluded at the time of last recording. CSS was calculated from the date of diagnosis to the date of cancer specific death. Deaths attributed to the lung cancer were treated as events and deaths from other causes were treated as censored observations. OS and CSS were estimated by the Kaplan-Meier (K-M) method and compared with the log-rank test. Multivariate Cox proportional hazard regression was used to determine 
independent prognostic factors. Hazard ratios (HRs) were calculated with $95 \%$ confidence intervals (CIs). Statistical analysis was performed with SPSS 24.0 (IBM Corp., Armonk, NY, USA), all statistical tests were two-sided, and $\mathrm{P}<0.05$ was considered statistically significant.

\section{Construction and validation of nomogram}

We assumed that patients received primary tumor resection that lived longer than median CSS time of those who didn't underwent surgery could benefit from the operation. According to this assumption, eligible patients who had undergone surgery to primary site were randomly divided into training and validation cohorts by 7:3. Both cohorts were then separately divided into two groups, beneficial group and non-beneficial group, according to the median CSS time of the non-surgery group (9 months, result from the cohort after PSM).

Factors that independently affect the CSS in multivariate Cox analysis and can be accessed before surgery were collected into the training set, including age, gender, TNM stage, histologic type, tumor position (over lapping lesion, bronchus tumor, lobe tumor) and differentiation grade. Based on the multivariable logistic analysis, we established the nomogram to predict the stage IV NSCLC candidates who can indeed benefit from primary site surgery in the training cohort. A nomogram was formulated by R software (version 2.15.1) with the rms package.

The areas under the ROC curve (AUC) and a calibration plots were derived based on the validation cohort by the regression analysis to assess the discriminative ability and accuracy of the nomograms, respectively.

According to the result of our predictive model, we divided all stage IV NSCLC patients in SEER database after PSM into three groups, one is beneficial \& surgery group whose assuming probability of benefit is over $50 \%$, and another is non-beneficial \& surgery group with less than $50 \%$ assuming probability of benefit, the other is non-surgery group. Kaplan-Meier analyses and log-rank test were conducted to test whether this model would distinguish patients that could benefit from primary tumor resection.

\section{Results}

\section{Patient characteristics before and after PSM}

A total of 565,424 lung cancer patients were identified in
SEER database from 2004 to 2014 and 30,342 stage IV NSCLC patients met the criteria (Figure 1). Of these eligible patients, 2,436 (8.03\%) underwent surgical treatment to primary tumor site. Obvious differences in age, gender, race, TNM stage, location, lesion laterality, histology, differentiation, radiation and distant surgery were noted between the two treatment groups before PSM (Table 1). Specifically, the surgery intervention was associated with small amount of older patient and higher TNM stage patients. This indicated that the baseline characteristics of the two groups (surgery and non-surgery) were not balanced. Variables that could influence the outcomes of treatment were included in 1: 1 PSM, including age, gender, TNM stage, differentiation grade, primary site, laterality, histological type, radiation therapy, chemotherapy and surgery to metastasis site.

After the 1:1 PSM, 4,872 stage IV NSCLC patients treated with or without primary site surgery were enrolled in the following survival analysis. Baseline characteristics, including age, gender, race, TNM stage, primary site, laterality, histology, differentiation, chemotherapy, radiation therapy and surgery to distant tumor, were all balanced $(\mathrm{P}>0.05)$, the detail is presented in Table 2.

\section{Impact of primary tumor resection on survival outcomes in stage IV NSCLC patients}

In the $\mathrm{K}-\mathrm{M}$ analyses and log-rank test in matched population, as seen in Table 3 and Figure 2, patients who underwent primary tumor resection enjoyed longer OS and CSS. The median CSS time was 19 months (95\% CI, 17.96-21.04) for patients who underwent primary tumor resection while only 9 months (95\% CI, 8.54-10.46) for patients who didn't receive surgery after PSM (HR $=0.47$, 95\% CI, 0.44-0.51, P<0.001). The 1 -year CSS rate was $31.1 \%$ and $50.9 \%$ for non-surgery and surgery groups, respectively. The 3 -year CSS rate was $7.1 \%$ and $20.3 \%$ for non-surgery and surgery patients after PSM (Table S1).

\section{Primary tumor resection as an independent prognostic factor for survival in stage IV NSCLC patients}

In multivariate Cox analysis (Table 2), primary tumor resection persisted to be independently associated with better OS (HR $=0.51,95 \% \mathrm{CI}, 0.47-0.55, \mathrm{P}<0.001)$ and CSS $(\mathrm{HR}=0.49,95 \% \mathrm{CI}, 0.46-0.52, \mathrm{P}<0.001)$ in matched population. Besides, age, gender, race, TNM stage, 


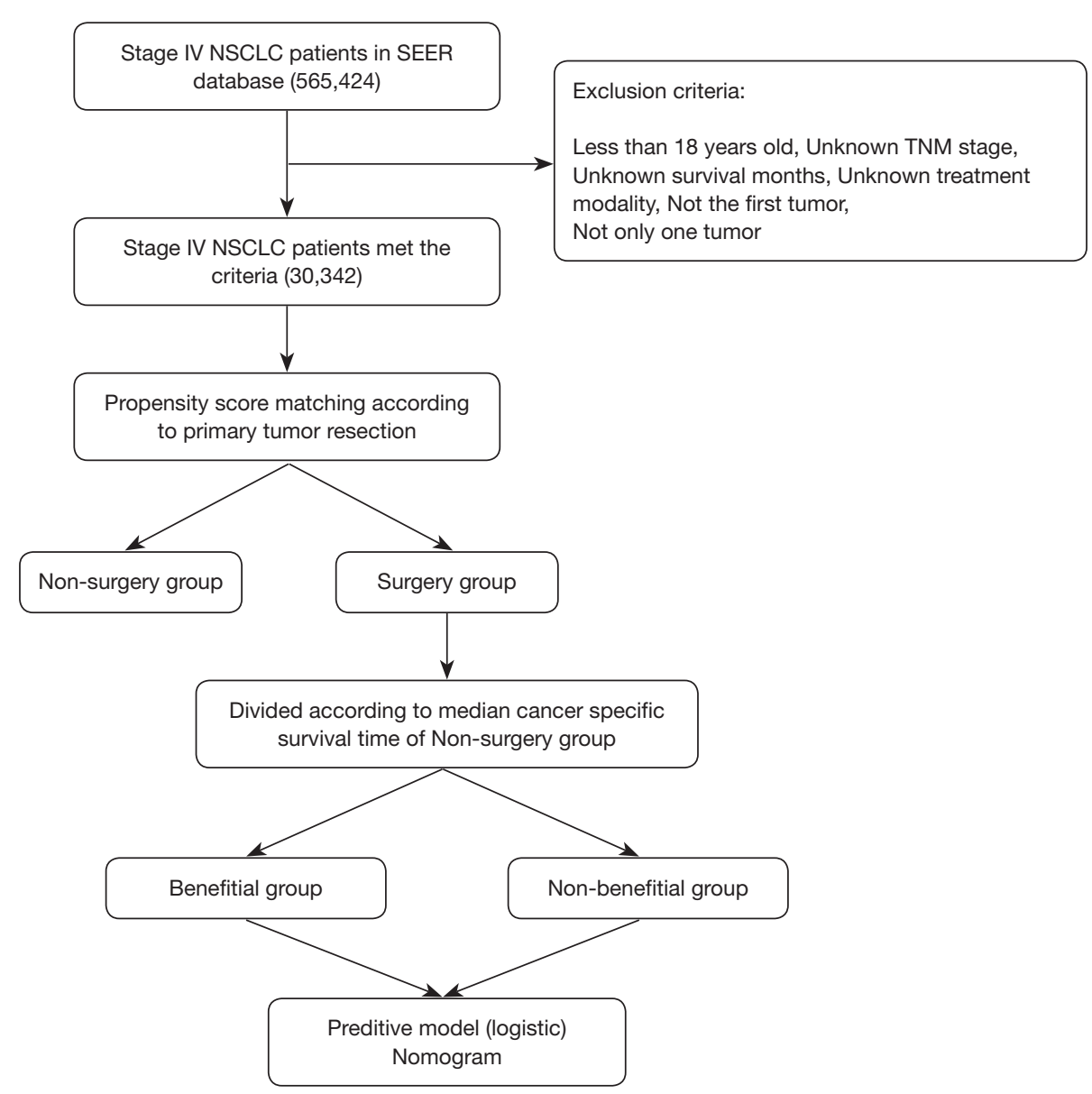

Figure 1 Flow chart of predictive model construction. SEER, Surveillance, Epidemiology and End Results; PSM, propensity score matching.

differentiation grade, primary site, chemotherapy and surgery to distant site are all independent factor for survival of stage IV NSCLC patients. Radiation and histology had no significant effect on overall survival and cancer specific survival.

\section{Nomogram to identify optimal candidates for primary tumor resection}

We assumed that patients received primary tumor surgery that lived longer than median CSS time (9 month) of these who didn't underwent surgery could benefit from the operation. Among the surgery cohort, 1,374 (56.40\%) patients lived longer than 9 months (beneficial group). According to this assumption, surgery receivers who survived longer than 9 months were classified into beneficial group and those who had shorter median CSS time (less than 9 months) were classified into non-beneficial group.

Based on beneficial and non-beneficial group, factors that independently affect the CSS in multivariate Cox analysis and can be accessed before surgery were collected into the training set, including six index: age, gender, TNM stage, histologic type, tumor position and differentiation grade. Based on the multivariate logistic regression, we established a nomogram to predict the stage IV NSCLC candidates who can indeed benefit from primary site surgery in the training cohort. A nomogram was then print (Figure 3). This model revealed that tumor position, age and differentiation contributed most to prognosis, followed by the $\mathrm{T}$ stage, $\mathrm{N}$ stage, histology, gender and $\mathrm{M}$ stage. By adding the scores of each selected variable, the likelihood of survival of the individual patient can be easily calculated. 
Table 1 Demographic information for patients with stage IV NSCLC before PSM

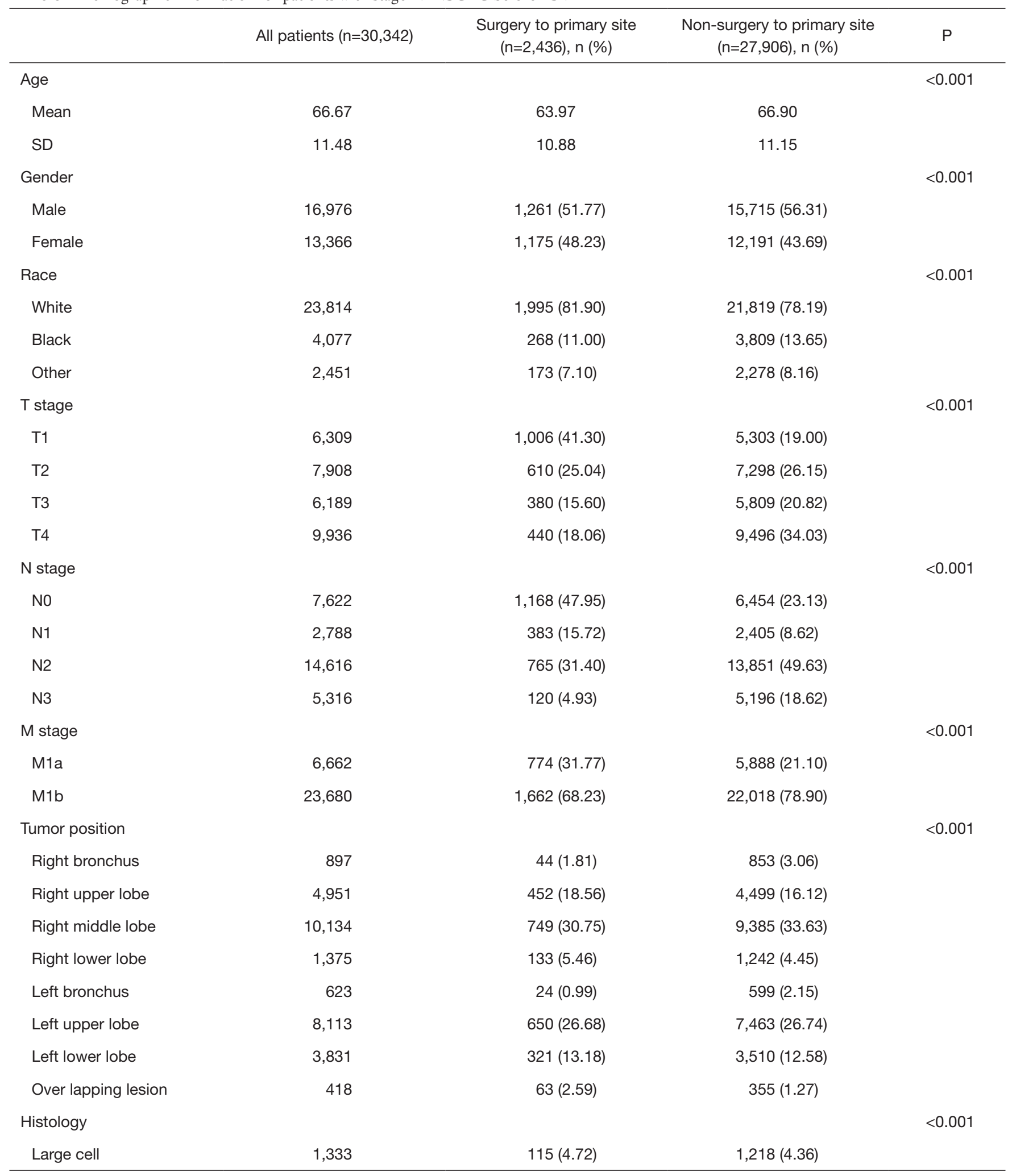

Table 1 (continued) 
Table 1 (continued)

\begin{tabular}{|c|c|c|c|c|}
\hline & All patients $(n=30,342)$ & $\begin{array}{l}\text { Surgery to primary site } \\
\qquad(n=2,436), n(\%)\end{array}$ & $\begin{array}{l}\text { Non-surgery to primary site } \\
\qquad(\mathrm{n}=27,906), \mathrm{n}(\%)\end{array}$ & $\mathrm{P}$ \\
\hline Adenocarcinoma & 18,573 & $1,649(67.69)$ & $16,924(60.65)$ & \\
\hline Squamous cell & 9,130 & $560(22.99)$ & $8,570(30.71)$ & \\
\hline Adeno-squamous & 517 & $80(3.28)$ & $437(1.57)$ & \\
\hline I & 1,789 & $195(8.00)$ & $1,594(5.71)$ & \\
\hline II & 9,184 & $904(37.11)$ & $8,280(29.67)$ & \\
\hline III & 18,164 & $1,240(50.90)$ & $16,924(60.65)$ & \\
\hline None/unknown & 14,892 & $1,349(55.38)$ & $13,543(48.53)$ & \\
\hline Chemotherapy & & & & 0.910 \\
\hline Yes & 16,832 & $1,354(55.58)$ & $15,478(55.46)$ & \\
\hline None/unknown & 13,510 & $1,082(44.42)$ & $12,428(44.54)$ & \\
\hline Surgery to other sites & & & & $<0.001$ \\
\hline Yes & 1,574 & $374(15.35)$ & $1,200(4.30)$ & \\
\hline None/unknown & 28,768 & $2,062(84.65)$ & $26,706(95.70)$ & \\
\hline
\end{tabular}

NSCLC, non-small cell lung cancer; PSM, propensity score matching; SD, standard deviation.

\section{Validation of the predictive model}

Internal validation via the training set indicated that the AUC index for the nomogram to predict CSS were 0.62 (95\% CI, 0.603-0.645), which was in agreement with actual CSS. External validation using the validation set, the AUC for the nomogram to predict CSS was 0.60 (95\% CI, 0.586-0.621) (Figure S1). The internal and external calibration plots of OS nomogram revealed a good correlation between prediction by the nomogram and actual observation (Figure S2).

We then validated the distinguish ability of the model in validation set. According to Kaplan-Meier analyses and log-rank test (Figure 4), beneficial \& surgery group is significantly live longer than non-beneficial \& surgery group $(\mathrm{HR}=0.57,95 \% \mathrm{CI}, 0.39-0.79, \mathrm{P}<0.001)$ or nonsurgery group $(\mathrm{HR}=0.54,95 \% \mathrm{CI}, 0.40-0.74, \mathrm{P}<0.001)$, but there no difference was observed between non-beneficial \& surgery and non-surgery group ( $\mathrm{HR}=0.97,95 \% \mathrm{CI}, 0.87$ 1.07, $\mathrm{P}=0.694)$.

\section{Clinical use}

To use the nomogram, first draw a vertical line to the top points row to assign points for each variable; then, add the points from each variable together and drop a vertical line from the total points row to obtain the probability of benefit from primary tumor resection (Figure 3). For example, for a 68 -year-old male patient with poorly differentiated bronchus adenocarcinoma which is classified as T3N2M1a stage, the total score is 220 points and the model predicts that the patients would benefit from primary tumor resection with the probability of $30 \%$.

Because the preoperative small specimen could not represent the whole tumor and the neoadjuvant therapy might alter tumor characteristics, not all cases represented accurate differentiation information. As a consequence, we constructed another model without differentiation information, the nomogram is presented in Figure S3. 
Table 2 Demographic information for patients with stage IV NSCLC after PSM

\begin{tabular}{|c|c|c|c|}
\hline & Surgery to primary site $(n=2,436), n(\%)$ & Non-surgery to primary site $(n=2,436), n(\%)$ & $\mathrm{P}$ \\
\hline Mean & 63.97 & 63.56 & \\
\hline SD & 10.88 & 11.02 & \\
\hline Gender & & & 0.566 \\
\hline Female & $1,175(48.23)$ & $1,189(48.81)$ & \\
\hline Race & & & 0.994 \\
\hline White & $1,995(81.90)$ & $1,994(81.80)$ & \\
\hline Black & $268(11.00)$ & $273(11.20)$ & \\
\hline $\mathrm{T} 1$ & $1,006(41.30)$ & $946(38.80)$ & \\
\hline $\mathrm{T} 2$ & $610(25.04)$ & $624(25.60)$ & \\
\hline T3 & $380(15.60)$ & $415(17.00)$ & \\
\hline $\mathrm{T} 4$ & $440(18.06)$ & $451(18.60)$ & \\
\hline $\mathrm{N}$ stage & & & 0.142 \\
\hline No & $1,168(47.95)$ & $1,158(47.60)$ & \\
\hline N1 & $383(15.72)$ & $345(14.20)$ & \\
\hline N2 & $765(31.40)$ & 785 (32.20) & \\
\hline Right bronchus & $44(1.81)$ & $43(1.77)$ & \\
\hline Right upper lobe & $452(18.56)$ & $460(18.88)$ & \\
\hline Right middle lobe & $749(30.75)$ & $744(30.54)$ & \\
\hline Right lower lobe & $133(5.46)$ & $129(5.30)$ & \\
\hline Left bronchus & $24(0.99)$ & $26(1.10)$ & \\
\hline Left upper lobe & $650(26.68)$ & $642(26.35)$ & \\
\hline Left lower lobe & $321(13.18)$ & $330(13.55)$ & \\
\hline Over lapping lesion & $63(2.59)$ & $56(2.20)$ & \\
\hline Histology & & & 0.878 \\
\hline Large cell & $115(4.72)$ & $105(4.40)$ & \\
\hline Adenocarcinoma & 1,649 (67.69) & $1,663(68.20)$ & \\
\hline
\end{tabular}

Table 2 (continued) 
Table 2 (continued)

\begin{tabular}{|c|c|c|c|}
\hline & Surgery to primary site $(n=2,436), n(\%)$ & Non-surgery to primary site $(n=2,436), n(\%)$ & $\mathrm{P}$ \\
\hline Adeno-squamous & $80(3.28)$ & $66(2.80)$ & \\
\hline Neuroendocrine & $32(1.31)$ & $35(1.40)$ & \\
\hline Grade & & & 0.492 \\
\hline II & $904(37.11)$ & $885(36.40)$ & \\
\hline III & $1,240(50.90)$ & $1,281(52.60)$ & \\
\hline IV & $97(3.98)$ & $97(4.00)$ & \\
\hline Radiation & & & 0.686 \\
\hline Chemotherapy & & & 0.437 \\
\hline Yes & $1,354(55.58)$ & $1,080(44.40)$ & \\
\hline None/unknown & $1,082(44.42)$ & $1,356(56.60)$ & \\
\hline Surgery to other sites & & & 0.469 \\
\hline Yes & $374(15.35)$ & $355(14.60)$ & \\
\hline None/unknown & $2,062(84.65)$ & $2,436(85.40)$ & \\
\hline
\end{tabular}

NSCLC, non-small cell lung cancer; PSM, propensity score matching; SD, Standard deviation.

Table 3 Multivariate Cox analysis for OS and CSS among PSM population

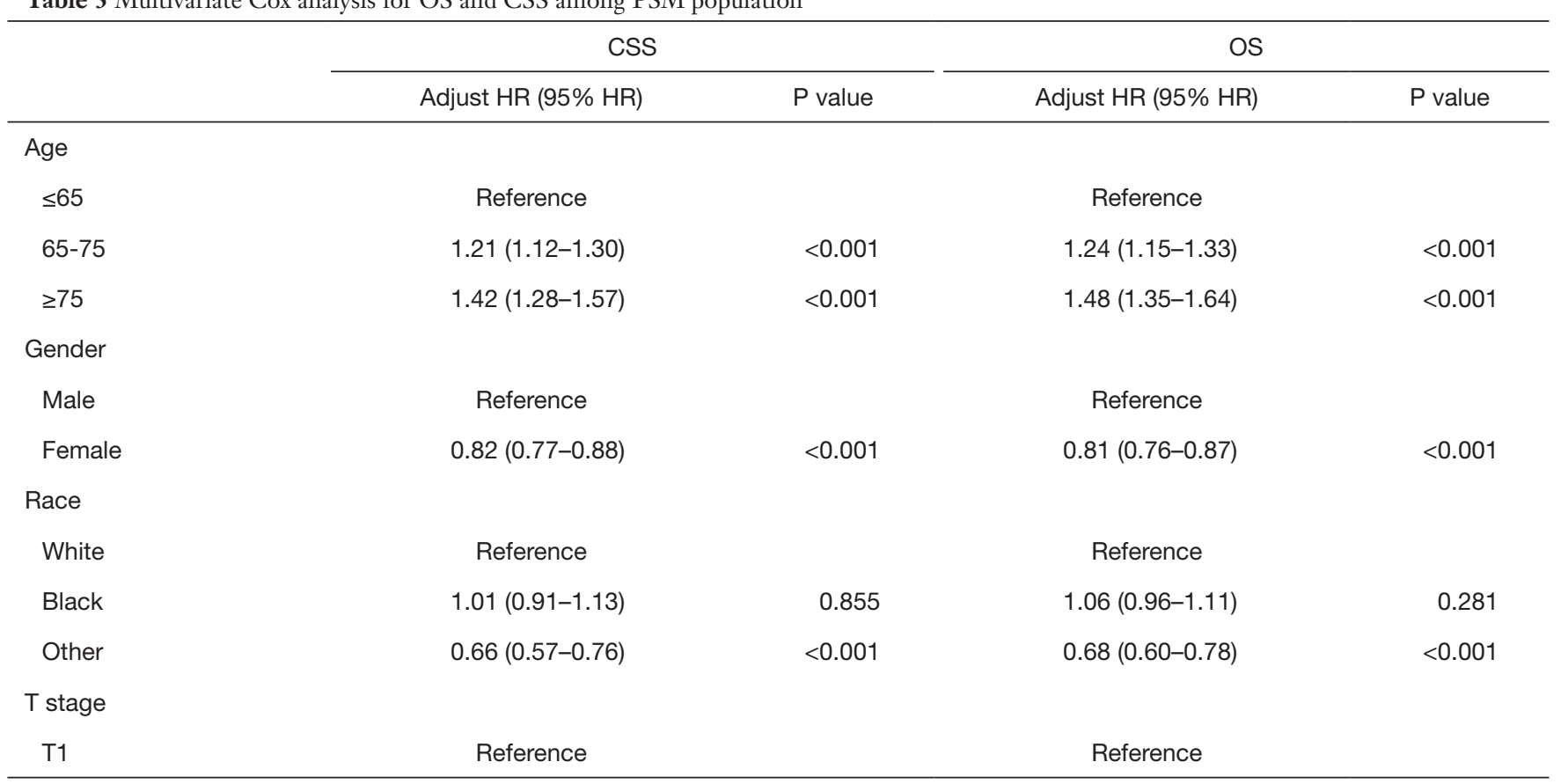

Table 3 (continued) 
Table 3 (continued)

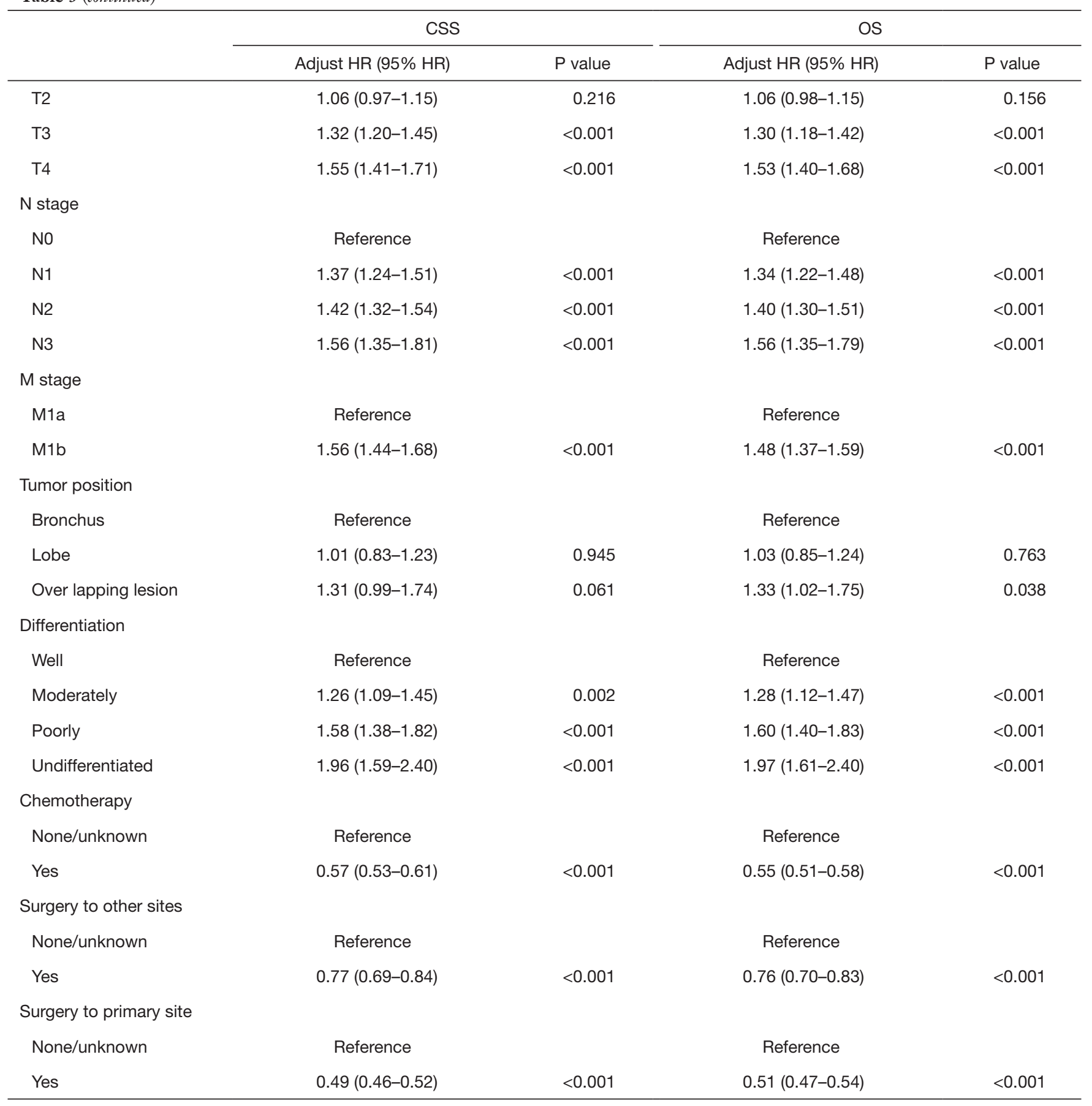

NSCLC, non-small cell lung cancer; PSM, propensity score matching; SD, standard deviation; OS, overall survival; CSS, cancer specific survival; HR, hazard ratio.

\section{Discussion}

These results confirmed our hypothesis and showed several important findings. Firstly, our study is in line with the previous reports $(5,7)$ that suggested a survival benefit of primary tumor resection for the stage IV in NSCLC patients. Secondly, different from the other study (13) which showed local consolidative therapy for patients with 
CSS of stage IV NSCLC Before PSM

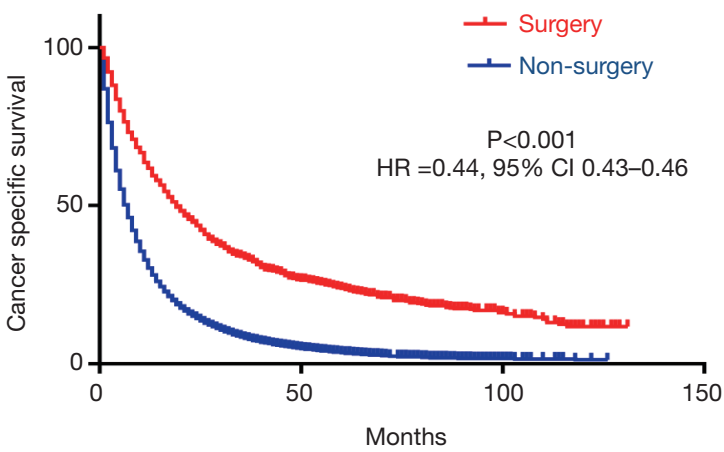

OS of stage IV NSCLC Before PSM

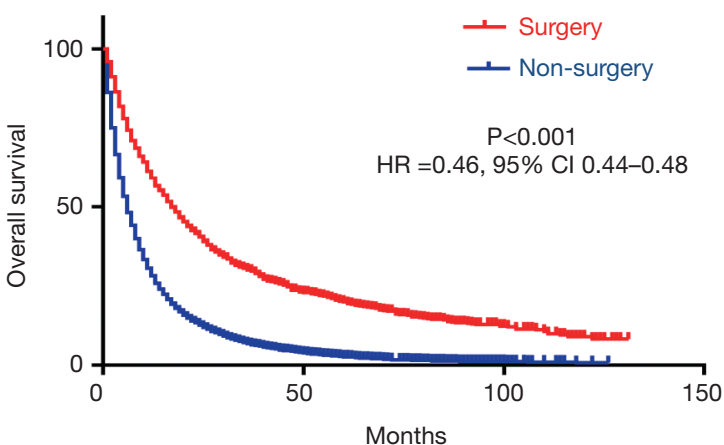

CSS of stage IV NSCLC after PSM

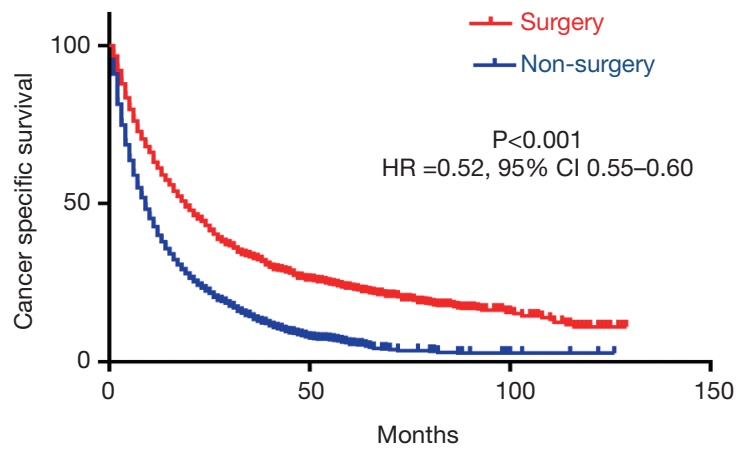

OS of stage IV NSCLC after PSM

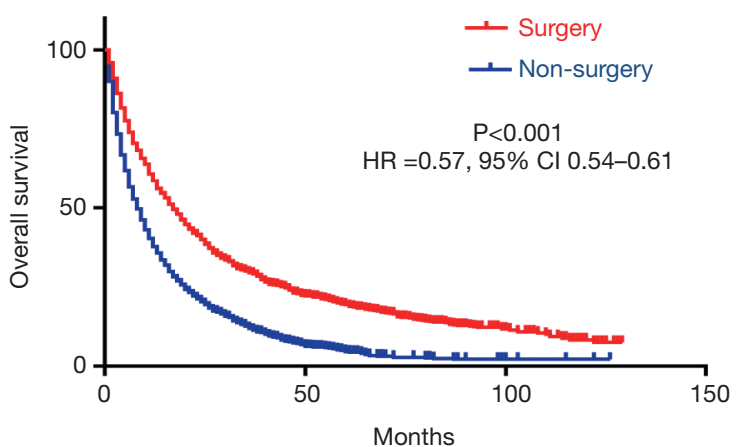

Figure 2 Kaplan-Meier plot of stage IV NSCLC patients according to treatment. NSCLC, non-small cell lung cancer; SEER, Surveillance, Epidemiology and End Results; PSM, propensity score matching; CSS, cancer specific survival; OS, overall survival; HR, hazard ratio.

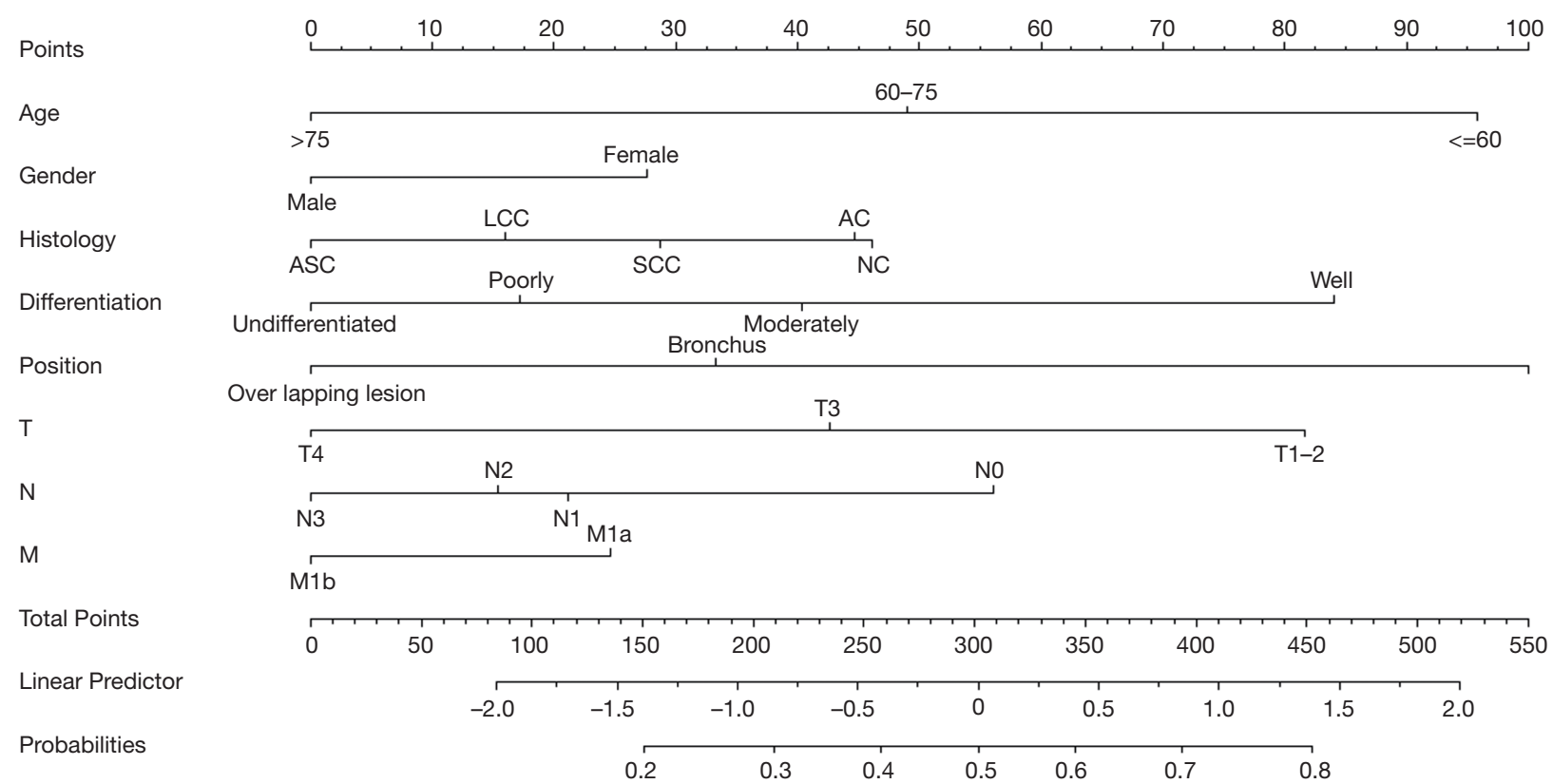

Figure 3 A nomogram to predict optimal candidates for primary tumor resection. ASC, adeno-squamous carcinoma; LCC, Large cell carcinoma; SCC, Squamous cell carcinoma; AC, adenocarcinoma; NC, Neuroendocrine. 


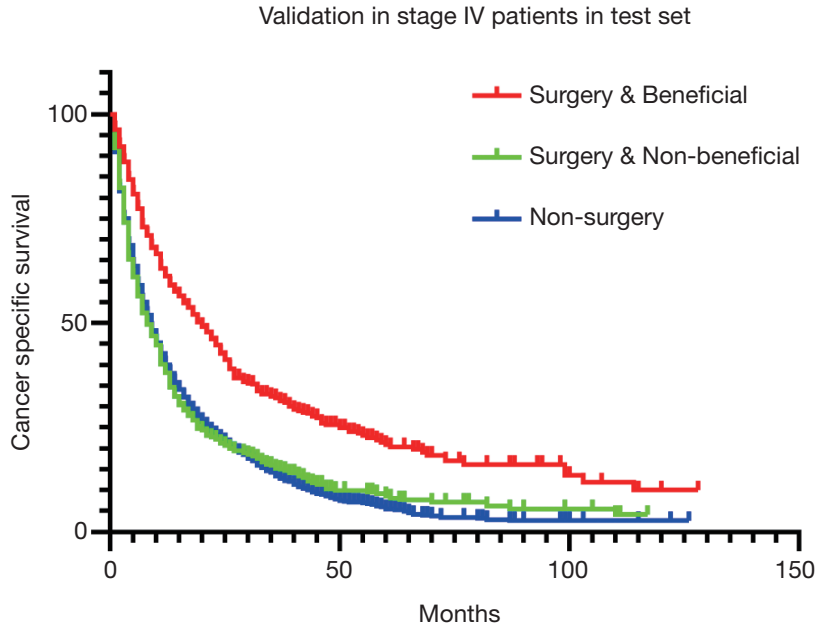

Figure 4 Kaplan-Meier plot to differentiate beneficial group according to our model in SEER database after PSM. SEER, Surveillance, Epidemiology and End Results; PSM, propensity score matching.

metastases NSCLC improved progression-free survival, we aimed to identify optimal candidates for surgery of the primary tumor and also try to comprehensively describe their clinical characters and power. Further, we created a visualized nomogram based on a logistic regression selective model to classify the optimal patients. Last, both internal and external validation demonstrated our model a useful and stable one.

In our model, lobe tumor position, younger age were the top 2 strongest predictors of patient who would be benefit from primary tumor resection. As is described before, smaller tumor lesion, less node invasion, and younger patients were associated with better outcomes for stage IV NSCLC patients who underwent primary tumor surgery (14), these facts indicated that self-conditions are crucial for surgery in advanced stage patients, which might owing to relative lower difficulty for surgery and thus with a longer life expectancy.

We came up with a new process to establish the decisionmaking model that pick the optimal patients who could indeed prolong their survival time via primary tumor surgery. Firstly, patients who underwent surgery and not were collected and matched according to baseline character to eliminate the selection bias. Secondly, median CSS time of surgery group and non-surgery group were calculated, we found after PSM, surgery group still own longer CSS time (19 vs. 9 months). Third, we divided the surgery patients into two group by the median CSS time of non- surgery patients ( 9 months), through which we believe that patients received primary tumor surgery that lived longer than 9 months could really benefit from the operation. Last, we compare the pre-operative baseline characters between surgery performed patients who live longer than 9 month (beneficial group) and who not (non-beneficial group), and then logistic model was used to construct the model. After such screening process, metastatic NSCLC patients who were really suitable for surgery were identified.

In 2017, Elizabeth A. David and colleagues develop a quantitative model predictive of selection for surgical treatment for patients with advanced-stage NSCLC (15). Using detailed clinical variables, readily available at the time of treatment decision making for advanced-stage NSCLC patients, they developed the SSS (a score system) that predicts the use of surgical intervention for these patients and found evidence that a therapeutic effect of resection remains after accounting for this SSS. Overall, AJCC metastatic status, AJCC nodal status, and age were the strongest predictors of patient selection for an operation in the SSS. While in our model, tumor position, age, differentiation and $\mathrm{T}$ stage were orderly indicated to be the most factor that could decide whether one could benefit from the primary site resection. The difference here demonstrated that patients who underwent primary resection might not be the optimal candidates, and also indicated the limitation of surgeons' recommendations.

In 2017, updates to the TNM staging system have reclassified metastatic NSCLC into M1a (separate tumor nodule in a contralateral lobe; tumor with pleural or pericardial nodules; or malignant pleural or pericardial effusion); M1b (single extra-thoracic metastasis in a single organ); or M1c (multiple extra-thoracic metastases in one or several organs) (16). We reclassified stage according to AJCC $8^{\text {th }}$ edition, but materials in SEER database were not enough to differentiate M1c. A study suggested that both M1b and M1c patients would benefit from local surgery (17). For patients with only one metastatic lesion, 5-year survival was approximately $50 \%$ after surgery, compared to only $17 \%$ for patients undergoing surgery with five metastatic lesions $(\mathrm{P}<0.001)$ (4). In our analysis, M1a patients seemed would gain more from primary tumor resection than $\mathrm{M} 1 \mathrm{~b}$, thus we have the reason to believe that M1c patients is not as suitable as M1b patients to receive surgery, systemic therapy was considered as priority in M1c patients.

We acknowledge some limitations in our study. First, the SEER database is lack of information on comorbidities that might lead to a selection bias for treatment choice. Second, 
the database also does not contain the number of metastatic sites, which have been shown to be an important predictors of surgery in IV stage patients (18). Third, data on systemic therapies were unavailable in SEER data base. The lack of information regarding target therapies and immunotherapy represents a major limitation of the current study. We are unable to answer whether before or after the systemic treatment would the primary tumor resection bring more survival profit. However, data from single center are limited and also lack of representative to evaluate such clinical question. SEER is the only comprehensive populationbased database and open access to worldwide represents an ideal approach to study the survival of patients in such setting.

\section{Conclusions}

We put forward a novel process to screening stage IV NSCLC patients who would really benefit from surgery. Our predictive model demonstrated that primary tumor resection had beneficial impact on metastatic NSCLC patients, and only specific patients would benefit from surgery and that the potential benefit would vary based on primary tumor characteristics. To be specified, in stage IV NSCLC patients, <60 years old, female, adenocarcinoma, well differentiation, tumor in lobe, T1-2, N0 and M1a NSCLC patients would be potentially benefit more from primary tumor resection. Once validated in a prospective cohort, our predictive model can be of great help in selecting the optimal candidates for primary tumor resection among metastatic NSCLC patients.

\section{Acknowledgments}

Funding: None.

\section{Footnote}

Reporting Checklist: The authors have completed the TRIPOD reporting checklist. Available at http://dx.doi. org/10.21037/tlcr-20-709

Peer Review File: Available at http://dx.doi.org/10.21037/ tlcr-20-709

Conflicts of Interest: All authors have completed the ICMJE uniform disclosure form (available at http://dx.doi. org/10.21037/tlcr-20-709). WL serves as an unpaid editorial board member of Translational Lung Cancer Research from Apr 2018 to Apr 2021. HL serves as an unpaid section editor of Translational Lung Cancer Research from Jan 2020 to Dec 2021.The other authors have no conflicts of interest to declare.

Etbical Statement: The authors are accountable for all aspects of the work in ensuring that questions related to the accuracy or integrity of any part of the work are appropriately investigated and resolved. The study was conducted in accordance with the Declaration of Helsinki (as revised in 2013).

Open Access Statement: This is an Open Access article distributed in accordance with the Creative Commons Attribution-NonCommercial-NoDerivs 4.0 International License (CC BY-NC-ND 4.0), which permits the noncommercial replication and distribution of the article with the strict proviso that no changes or edits are made and the original work is properly cited (including links to both the formal publication through the relevant DOI and the license). See: https://creativecommons.org/licenses/by-nc-nd/4.0/.

\section{References}

1. Torre LA, Siegel RL, Jemal A. Lung Cancer Statistics. Adv Exp Med Biol 2016;893:1-19.

2. Siegel RL, Miller KD, Jemal A. Cancer Statistics, 2017. CA Cancer J Clin 2017;67:7-30.

3. Hofman P. First-line immunotherapy for patients with advanced stage or metastatic non-small cell lung cancer finally what threshold of PD-L1 expression on tumor cells? Transl Lung Cancer Res 2019;8:728-30.

4. Hanagiri T, Takenaka M, Oka S, et al. Results of a surgical resection for patients with stage IV non--small-cell lung cancer. Clin Lung Cancer 2012;13:220-4.

5. David EA, Canter RJ, Chen Y, et al. Surgical Management of Advanced Non-Small Cell Lung Cancer Is Decreasing But Is Associated With Improved Survival. Ann Thorac Surg 2016;102:1101-9.

6. Kawano D, Takeo S, Katsura M, et al. Surgical treatment of stage IV non-small cell lung cancer. Interact Cardiovasc Thorac Surg 2012;14:167-70.

7. Abdel-Rahman O. Outcomes of Surgery as Part of the Management of Metastatic Non-Small-Cell Lung Cancer: A Surveillance, Epidemiology and End Results Database Analysis. Cancer Invest 2018;36:238-45.

8. Liu K, Zheng D, Xu G, et al. Local thoracic therapy 
improve prognosis for stage IV non-small cell lung cancer patients combined with chemotherapy: A Surveillance, Epidemiology, and End Results database analysis. PLoS One 2017;12:e0187350.

9. Sun Z, Sui X, Yang F, et al. Effects of primary tumor resection on the survival of patients with stage IV extrathoracic metastatic non-small cell lung cancer: A population-based study. Lung Cancer 2019;129:98-106.

10. Li H, Sun Z, Yang F, et al. Primary tumour resection in non-small-cell lung cancer patients with ipsilateral pleural dissemination (M1a): a population-based study. Eur J Cardiothorac Surg 2019;55:1121-9.

11. Collins GS, Reitsma JB, Altman DG, et al. Transparent Reporting of a multivariable prediction model for Individual Prognosis or Diagnosis (TRIPOD): the TRIPOD Statement. Br J Surg 2015;102:148-58.

12. Doll KM, Rademaker A, Sosa JA. Practical Guide to Surgical Data Sets: Surveillance, Epidemiology, and End Results (SEER) Database. JAMA Surg 2018;153:588-9.

13. Gomez DR, Blumenschein GR Jr, Lee JJ, et al. Local consolidative therapy versus maintenance therapy or observation for patients with oligometastatic non-small-

Cite this article as: Liang $\mathrm{H}$, Liu Z, Huang J, Liu J, Wang W, Li J, Xiong S, Li C, Cheng B, Zhao Y, Cui F, He J, Liang W. Identifying optimal candidates for primary tumor resection among metastatic non-small cell lung cancer patients: a population-based predictive model. Transl Lung Cancer Res 2021;10(1):279-291. doi: 10.21037/tlcr-20-709 cell lung cancer without progression after first-line systemic therapy: a multicentre, randomised, controlled, phase 2 study. Lancet Oncol 2016;17:1672-82.

14. Yang CJ, Gu L, Shah SA, et al. Long-term outcomes of surgical resection for stage IV non-small-cell lung cancer: A national analysis. Lung Cancer 2018;115:75-83.

15. David EA, Andersen SW, Beckett LA, et al. A Model to Predict the Use of Surgical Resection for Advanced-Stage Non-Small Cell Lung Cancer Patients. Ann Thorac Surg 2017;104:1665-72.

16. Shin J, Keam B, Kim M, et al. Prognostic Impact of Newly Proposed M Descriptors in TNM Classification of NonSmall Cell Lung Cancer. J Thorac Oncol 2017;12:520-8.

17. Nieder C, Hintz M, Oehlke O, et al. The TNM 8 M1b and M1c classification for non-small cell lung cancer in a cohort of patients with brain metastases. Clin Transl Oncol 2017;19:1141-6.

18. Zhang T, Zhang JT, Li WF, et al. Visceral pleural invasion in $\mathrm{T} 1$ tumors $(3 \mathrm{~cm})$, particularly T1a, in the eighth tumor-node-metastasis classification system for non-small cell lung cancer: a population-based study. J Thorac Dis 2019;11:2754-62. 
A

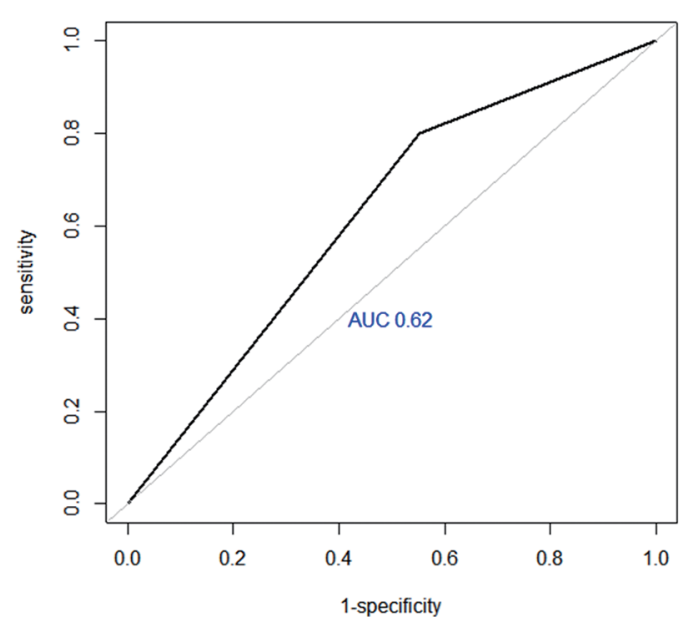

B

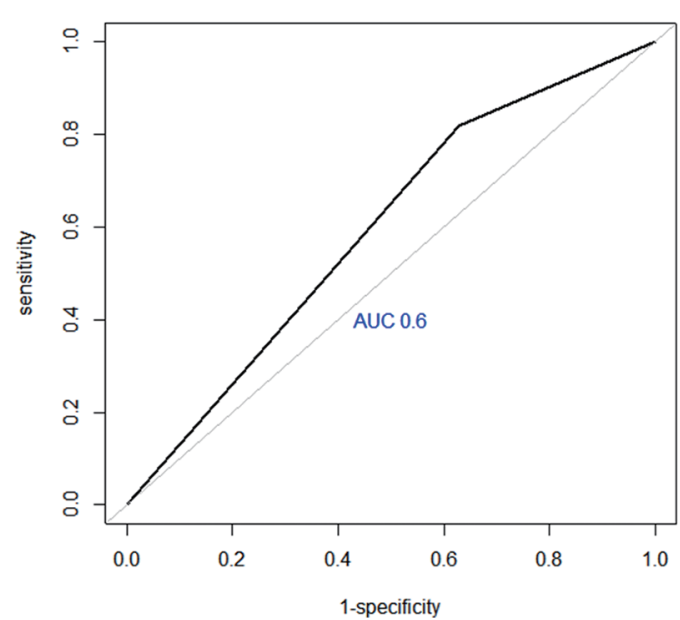

Figure S1 Receiver operating characteristic curve of this model (A) Internal validation; (B) External validation. AUC, areas under the ROC curve.

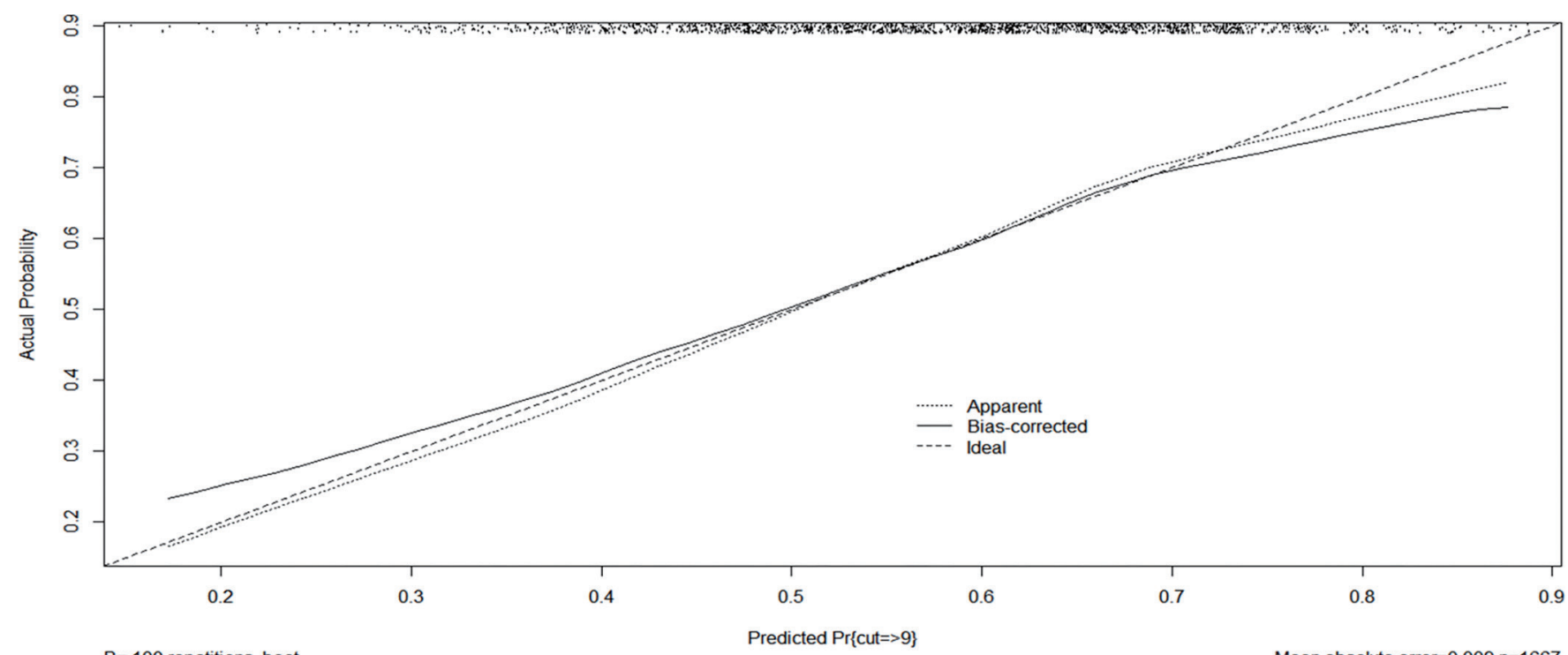

$B=100$ repetitions, boot

Mean absolute error $=0.009 n=1667$

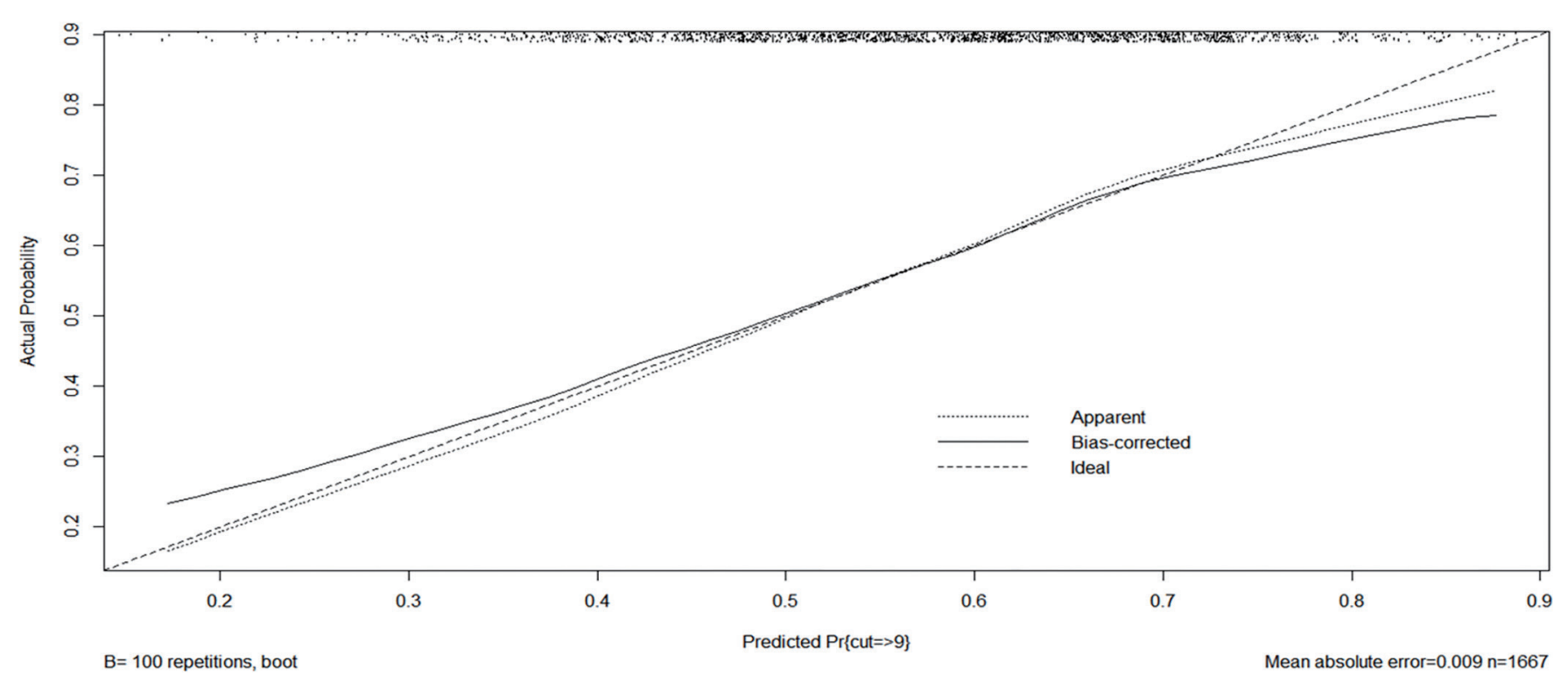

Figure S2 The calibration curve for predicting survival benefit from surgery of stage IV NSCLC patient. NSCLC, non-small cell lung cancer. 
Points

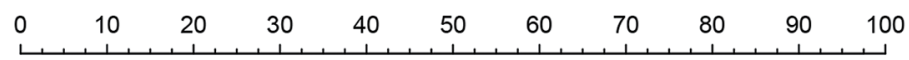

Age

Gender

Histology

Position

T

$\mathrm{N}$

M

Total Points

Linear Predictor

Probabilities
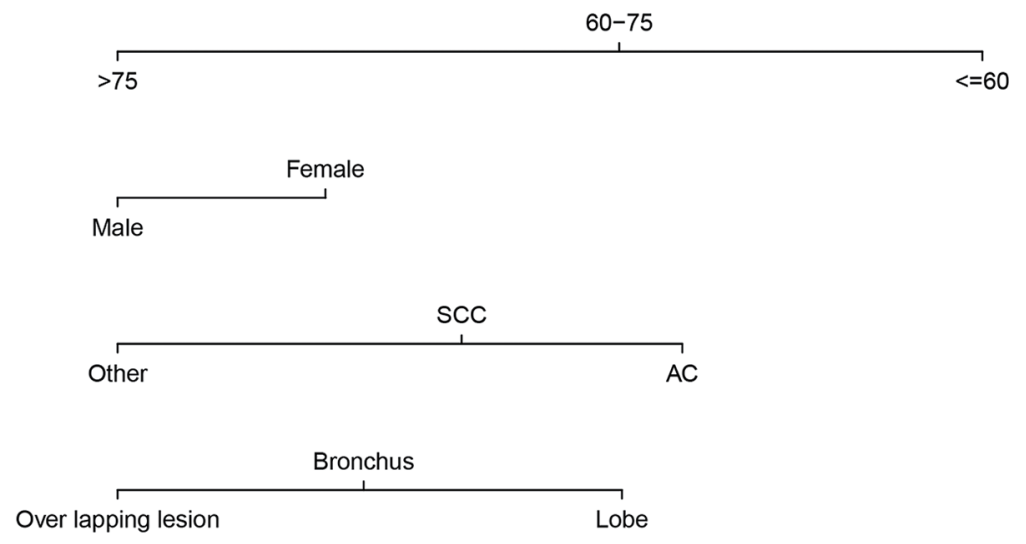

T3
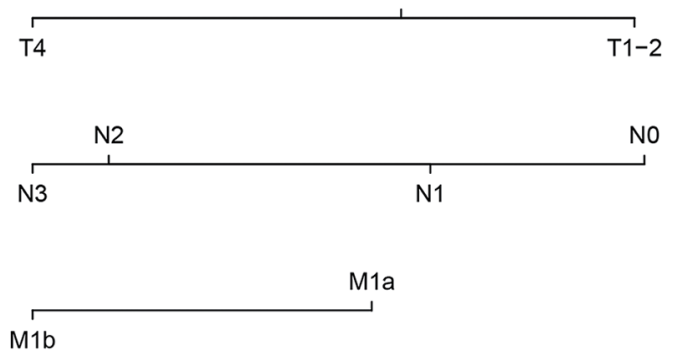

M1a

M1b
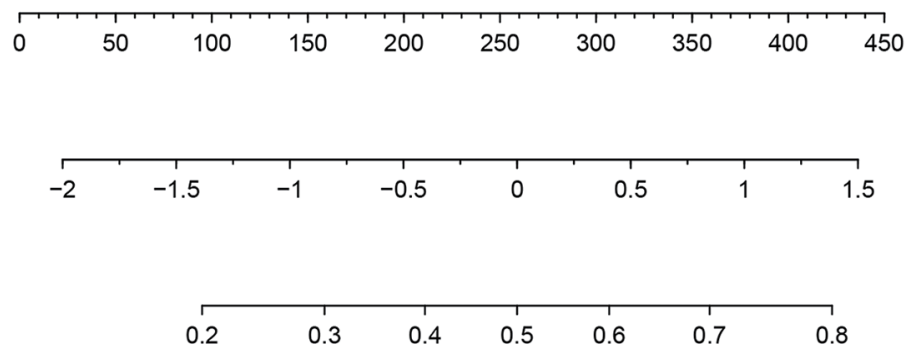

Figure S3 An adjusted nomogram to predict optimal candidates for primary tumor resection. SCC, squamous cell carcinoma; AC, adenocarcinoma.

Table S1 Median survival time of patients according to treatment

\begin{tabular}{lcccccc}
\hline & \multicolumn{2}{c}{ Before PSM } & & \multicolumn{2}{c}{ After PSM } \\
\cline { 2 - 3 } & Surgery vs. non-surgery (HR; 95\% Cl) & P value & & Surgery vs. non-surgery (HR; 95\% Cl) & P value \\
\hline Median OS & 17 vs. 6 $(0.35 ; 0.34-0.37)$ & $<0.001$ & & 17 vs. $8(0.47 ; 0.44-0.50)$ & $<0.001$ \\
Median CSS & 19 vs. $7(0.37 ; 0.35-0.39)$ & $<0.001$ & & 19 vs. 9 $(0.47 ; 0.44-0.51)$ & $<0.001$ \\
\hline
\end{tabular}

